\title{
Other and III Defined Digestive Organs ICD-O-3
}

National Cancer Institute

\section{Source}

National Cancer Institute. Other and III Defined Digestive Organs ICD-O-3. NCI

Thesaurus. Code C148335.

Digestive organs that are referenced in topographic category C26 of ICD-O-3. 